\title{
Effects of novel synthetic serine protease inhibitors on postoperative blood loss, coagulation parameters, and vascular relaxation after cardiac surgery
}

\author{
Gábor Szabó, MD, PhD, ${ }^{\mathrm{a}}$ Gábor Veres, MD, ${ }^{\mathrm{b}}$ Tamás Radovits, MD, ${ }^{\mathrm{a}}$ Humaira Haider, MD, ${ }^{\mathrm{a}}$ \\ Nelli Krieger, MD, ${ }^{\mathrm{a}}$ Susanne Bährle, MD, ${ }^{\mathrm{c}}$ Christiane Miesel-Gröschel, MTA, ${ }^{\mathrm{a}}$ Silke Niklisch, MTA, ${ }^{\mathrm{d}}$ \\ Matthias Karck, MD, ${ }^{\mathrm{a}}$ and Andreas van de Locht, $\mathrm{PhD}^{\mathrm{d}}$
}

\begin{abstract}
Objective: Although aprotinin has been widely used to reduce perioperative blood loss after cardiopulmonary bypass, recent concerns have led to its withdrawal. This study investigated effects of the novel synthetic serine protease inhibitors CU-2010 and CU-2020 on blood loss, coagulation parameters, and coronary relaxation in a canine model.

Methods: Thirty-seven dogs were divided into 5 groups: control $(n=5)$, aprotinin $(n=8$, Hammersmith scheme of intravenous bolus, prime, and continuous infusion), Hammersmith CU-2010 ( $\mathrm{n}=8,1.6 \mathrm{mg} / \mathrm{kg}$ Hammersmith scheme), continuous CU-2010 ( $\mathrm{n}=8,1.6 \mathrm{mg} / \mathrm{kg}$ continuous infusion), and CU-2020 ( $\mathrm{n}=8,8.9 \mathrm{mg} / \mathrm{kg}$ Hammersmith scheme). All animals underwent 90 -minute cardiopulmonary bypass. End points were blood loss during first 2 hours after protamine and activated clotting, partial thromboplastin, and prothrombin times. At end of experiments, coronary rings were removed for in vitro testing of relaxation to acetylcholine and sodium nitroprusside.

Results: Hammersmith and continuous CU-2010, CU-2020, and aprotinin groups all had reduced blood loss $(43 \pm 4,43 \pm 8,52 \pm 7,61 \pm 7$, respectively, vs control $149 \pm 24 \mathrm{~mL}, P<.05)$. After protamine, activated clotting time and partial thromboplastin time normalized in control, aprotinin, and Hammersmith CU-2010 groups but remained elevated in continuous CU-2010 and CU-2020 groups. Prothrombin time and vascular relaxation did not differ between groups.
\end{abstract}

Conclusions: CU-2010 and CU-2020 significantly reduced blood loss after cardiac surgery, with prolonged partial thromboplastin and activated clotting times, demonstrating improved antithrombotic profile. Neither aprotinin nor the novel serine protease inhibitors influenced vascular relaxation. (J Thorac Cardiovasc Surg 2010;139:181-8)

Excessive postoperative bleeding during cardiac surgery occurs in $3.6 \%$ of patients undergoing coronary artery bypass grafting and increases to $11 \%$ among those requiring more complex operations. ${ }^{1,2}$ Reoperation for bleeding increases hospital mortality 3 - to 4 -fold, ${ }^{1,2}$ substantially lengthens postoperative hospital stay, and has a sizeable effect on health care costs. Even without the need for reoperation, blood loss frequently leads to transfusion of allogeneic blood products, which exposes patients to risks of transfusion-related adverse effects, including allergic reactions, transfusion errors, and blood-borne infections. Concerns

\footnotetext{
From the Departments of Cardiac Surgery ${ }^{\mathrm{a}}$ and Cardiology, Angiology, and Pulmonology, ${ }^{\mathrm{b}}$ University of Heidelberg, Heidelberg, Germany; the Department of Cardiovascular Surgery, Semmelweis University Medical School, Budapest, Hungary ${ }^{c}$; and Curacyte Discovery GmbH, Leipzig, Germany. ${ }^{\mathrm{d}}$

Read at the Eighty-eighth Annual Meeting of The American Association for Thoracic Surgery, San Diego, Calif, May 10-14, 2008.

Disclosures: Andreas van de Locht is an employee of Curacyte GmbH; Gábor Szabó reports consulting fees and grant support for this study from Curacyte Ltd, the manufacturer of the analogues.

Supported by research grant from Curacyte Discovery GmbH, Leipzig, Germany.

Received for publication May 8, 2008; revisions received Aug 9, 2009; accepted for publication Sept 7, 2009.

Address for reprints: Gábor Szabó, MD, PhD, Department of Cardiac Surgery, Im Neuenheimer Feld 110,69120 Heidelberg, Germany (E-mail: gabor.szabo@urz. uni-heidelberg.de).

$0022-5223 / \$ 36.00$

Copyright (C) 2010 by The American Association for Thoracic Surgery

doi:10.1016/j.jtcvs.2009.09.019
}

about transfusion safety, blood product shortages, and increasing blood bank costs have generated growing interest in risk-reduction strategies for postoperative bleeding.

The nonspecific serine protease inhibitor aprotinin was formerly used to reduce the perioperative blood loss and the need for blood transfusion after cardiopulmonary bypass (CPB). Aprotinin is produced from bovine tissue, so the possibility of an allergic reaction must be considered whenever this drug is used. The anaphylactic potential of aprotinin has been a major concern; the overall risk of anaphylaxis to aprotinin is estimated to be $0.5 \%$, and even higher in reexposed patients at approximately $2.8 \%{ }^{3}$ Allergic or severe anaphylactic reactions have been reported, ${ }^{4}$ although mainly with reexposure. Because aprotinin is a bovine protein, the possibility of an infectious disease is also raised. These concerns have led to reduced use of aprotinin. Novel serine protease inhibitors have been in development for the following reasons: (1) to simplify drug synthesis (aprotinin is isolated from bovine lungs in a costly procedure), (2) to avoid the allergic potential of the commercial aprotinin product (which contains additional proteins that must be considered whenever this drug is used), and (3) to avoid the theoretic infectious risks of bovine protein products. The compounds tested in this study were developed to address these issues while aiming at comparable blood loss reduction to aprotinin. 


$$
\begin{aligned}
& \text { Abbreviations and Acronyms } \\
& \begin{aligned}
& \mathrm{ACT}=\text { activated clotting time } \\
& \mathrm{aPTT}=\text { activated partial thromboplastin time } \\
& \mathrm{CPB}=\text { cardiopulmonary bypass } \\
& \mathrm{EC}_{200}= \text { Concentration for } 2 \text {-fold prolongation of } \\
& \text { clotting time } \\
& \mathrm{K}_{\mathrm{i}}=\text { affinity constant } \\
& \mathrm{KIU}=\text { kallikrein inactivator units } \\
& \mathrm{PT}=\text { prothrombin time } \\
& \mathrm{SNP}=\text { sodium nitroprusside }
\end{aligned}
\end{aligned}
$$

CU-2010 (Curacyte Discovery GmbH, Leipzig, Germany) is a novel, small, synthetic compound (molecular weight $700 \mathrm{Da}$ ) with peptidelike characteristics that allow substratelike binding to the active site of serine proteases (Katz and colleagues ${ }^{5}$ reviewed this class of compounds). CU-2010 shows rapid clearance after intravenous infusion, with an elimination half-life of approximately 20 minutes in rats and dogs (unpublished animal experiment data, Nicklisch S, Curacyte Discovery, January 2007). CU-2020 is the polymerized and pegylated form of CU-2010 with a prolonged half-life (45 minutes).

In the past, our group has been involved in the development of novel synthetic small-molecule serine protease inhibitors. The potential advantage of this new class of serine protease inhibitors includes the elimination of allergic and infectious risks and the reduction of thromboembolic risk through improved antifibrinolytic and anticoagulatory profiles. The primary aim of this study was to compare the efficacy of the new synthetic serine protease inhibitors CU-2010 and CU-2020 on curtailing blood loss with that of bovine aprotinin in a canine model of extracorporeal circulation. Special interest was focused on the issue whether a simplified dosing scheme (simple continuous infusion) would lead to the same effectiveness as the combination of boluses and intravenous infusion.

Endothelial function and thrombus formation are closely related. Injury of the endothelial surface leads to increased rate of thrombus formation. Endothelial integrity is also a key factor in regulation of blood flow and in defense against inflammation or ischemia-reperfusion injury. The vascular effects of aprotinin have only been partially clarified. Many authors have shown that aprotinin causes graft occlusion, especially venous graft occlusion ${ }^{6,7}$; however, no clinical association between aprotinin use and graft occlusion could be found in other studies. ${ }^{8}$ Experimental studies have resulted in conflicting data. Khan and colleagues ${ }^{9}$ have described favorable effects of aprotinin on endothelial function in experimental model. There have been also some experimental observations that aprotinin impairs endothelium-dependent relaxation. ${ }^{10,11}$ The secondary aim of our experiment was therefore to examine the effects of aprotinin analogs on endothelium-dependent and -independent vasorelaxation of coronary arteries and on coagulation parameters.

\section{MATERIALS AND METHODS \\ In Vitro Measurements}

Serine protease inhibitory activities and corresponding affinity constant $\left(\mathrm{K}_{\mathrm{i}}\right)$ values were determined by standard enzyme kinetic assays and were provided by the manufacturer (Curacyte Discovery; Tables 1 and 2).

Prothrombin time (PT) and activated partial thromboplastin time (aPTT) were determined after standard human plasma (Dade Behring, Eschborn, Germany) was supplemented with test compound solution or saline solution with a coagulation analyzer (Sysmex CA-560; Dade Behring) according to manufacturer instructions. Reagents used were Innovin for PT and Actin FSL for aPTT, both from Dade Behring. Aprotinin was purchased from Sigma (Sigma, St Louis, Mo). From each concentration-response curve, $\mathrm{EC}_{200}$ values, defined as the concentration required for 2-fold prolongation of clotting time, were calculated.

\begin{abstract}
Animals
All animals received humane care and treatment in compliance with the "Principles of Laboratory Animal Care" formulated by the National Society for Medical Research and in accordance with the "Guide for the Care and Use of Laboratory Animals" (www.nap.edu/catalog/5140.html). Power analysis to determine the necessary number of animals was based on the primary end point of blood loss. We accepted an $\alpha$ error of .05 and a $\beta$ error of .15. Taking dropouts into account, 10 animals per group were required for this study. The experiments were approved by the ethical committee of the Land Baden-Württemberg, Germany, for Animal Experimentation. All together, $37 \mathrm{dogs}$ (foxhounds) weighing 20 to $35 \mathrm{~kg}$ were used in these experiments.
\end{abstract}

\section{Surgical Preparation and General Management}

The dogs were medicated in advance with propionylpromazine and anesthetized with a bolus of pentobarbital $(15 \mathrm{mg} / \mathrm{kg}$ initial bolus and then 0.5 $\mathrm{mg} /[\mathrm{kg} \cdot \mathrm{h}]$ intravenously), paralyzed with pancuronium bromide $(0.1$ $\mathrm{mg} / \mathrm{kg}$ as a bolus and then $0.2 \mathrm{mg} /[\mathrm{kg} \cdot \mathrm{h}]$ intravenously), and endotracheally intubated. The dogs were ventilated with a mixture of room air and oxygen (inspired fraction of oxygen $60 \%$ ) at a frequency of 12 to 15 breaths $/ \mathrm{min}$ and a tidal volume starting at $15 \mathrm{~mL} /(\mathrm{kg} \cdot \mathrm{min})$. The settings were adjusted by maintaining arterial $\mathrm{PCO}_{2}$ between 35 and $40 \mathrm{~mm} \mathrm{Hg}$. The femoral artery and vein were cannulated to record mean arterial pressure and take blood samples for the analysis of blood gases, electrolytes, $\mathrm{pH}$, and parameters of blood coagulation. Basic intravenous volume substitution was carried out with Ringer's solution at a rate of $1 \mathrm{~mL} /(\mathrm{kg} \cdot \mathrm{min})$. If necessary, the rate of volume substitution was modified according to the continuously controlled input-output balance to maintain hemodynamic stability. According to the values of potassium, bicarbonate, and base excess, substitution included administration of potassium chloride and sodium bicarbonate $(8.4 \%)$. Neither catecholamines nor other hormonal or pressor substances were administered. Rectal temperature and standard peripheral electrocardiogram were monitored continuously.

After left anterolateral thoracotomy in the fourth intercostal space, pericardiotomy, and isolation of the great vessels, a perivascular ultrasonic flow probe was attached to the ascending aorta. Aortic pressure was monitored with a 5F Millar catheter tip manometer (Millar Instruments Inc, Houston, Tex).

\section{Cardiopulmonary Bypass}

After systemic anticoagulation with sodium heparin $(300 \mathrm{U} / \mathrm{kg})$, the left subclavian artery was cannulated for arterial perfusion. The venous cannula 
TABLE 1. Inhibition of human serine proteases

\begin{tabular}{lccc}
\hline & CU-2010 & CU-2020 & Aprotinin \\
\hline Plasmin & 2.2 & 8.4 & 4.2 \\
Plasma kallikrein & 0.04 & 0.16 & 31 \\
Factor Xa & 51 & 81 & 218,000 \\
Factor XIa & 26 & 819 & 2687 \\
Thrombin & 1423 & 1553 & 164,000 \\
Factor XIIa & 4040 & 892 & 2951 \\
\hline
\end{tabular}

Values are given in nanomoles per liter.

was placed in the right atrium. The extracorporeal circuit consisted of a heat exchanger, a venous reservoir, a roller pump, and a membrane oxygenator primed with Ringer's lactate solution $(1000 \mathrm{~mL})$ supplemented with heparin $(150 \mathrm{U} / \mathrm{kg}$ ) and $20 \mathrm{~mL}$ sodium bicarbonate $(8.4 \%)$. Normothermic CPB was performed for 90 minutes. After weaning from CPB, heparin was antagonized with protamine administered intravenously during the course of 10 minutes, and the animals were monitored for 2 hours. Hearts and coronary arteries were then excised for further investigation.

\section{Experimental Groups}

Thirty-seven dogs were divided into 5 experimental groups: control animals $(n=5)$ received placebo, the aprotinin treatment group $(n=8)$ received bovine aprotinin, and the remaining dogs received the new synthetic aprotinin analogs CU-2010 ( 2 treatment schemes, $\mathrm{n}=8$ each) and CU-2020 $(n=8)$. In the bovine-derived aprotinin group, the treatment was applied according to the Hammersmith scheme (intravenous bolus, pump prime, and continuous infusion) for a body weight of $30 \mathrm{~kg}$, starting with an intravenous bolus ( 0.85 million kallikrein inactivator units [KIU]) just before $\mathrm{CPB}$ was initiated, followed by a pump prime $(0.85$ million $\mathrm{KIU}$ ) and an infusion during $\mathrm{CPB}$ at a rate of 0.2 million KIU/h for 90 minutes ( 0.3 million KIU total). The applied dose of aprotinin was adjusted to the actual body weight of each animal. Accordingly, the animals received 28,333 KIU/kg as an initial bolus and 28,333 KIU/ $/ \mathrm{kg}$ in the pump prime. During $\mathrm{CPB}, 10,000 \mathrm{KIU} / \mathrm{kg}$ aprotinin was infused during 90 minutes. Dogs receiving the aprotinin analog treatments were divided three groups: the Hammersmith CU-2010 group received a $0.8 \mathrm{mg} / \mathrm{kg}$ bolus before CPB, followed by a pump prime $(0.4 \mathrm{mg} / \mathrm{kg})$ and a continuous infusion $(0.4 \mathrm{mg} / \mathrm{kg}$ ) during CPB (90 minutes); the continuous CU-2010 group received $1.6 \mathrm{mg} / \mathrm{kg}$ continuous infusion during the experiment (220 minutes); and the CU-2020 group received a $1.66 \mathrm{mg} / \mathrm{kg}$ bolus before $\mathrm{CPB}$, followed by a pump prime $(1.66 \mathrm{mg} / \mathrm{kg})$ and $4.98 \mathrm{mg} / \mathrm{kg}$ infused during 90 minutes of CPB. The doses were selected primarily on the basis of in vitro data, aiming for a dose more or less equipotential with aprotinin. Furthermore, the observed half-life values (CU-2010 17 minutes, CU-2020 45 minutes) were taken into account in the selection of the doses.

\section{Measurements of Blood Loss and Biochemical and Hemodynamic Parameters}

The primary end point of the study was the total blood loss after weaning from CPB during the first 2 hours after application of protamine. Blood loss was measured from gauze bandages after weaning from CPB and 15 minutes after finishing of protamine at different time points $(120,160$, and 220 minutes after initiation of CPB). Bandages were placed into the operating area (pericardial sac and surrounding tissues). Weight of gauze bandages was measured before and after cleaning of the operating area. We calculated blood loss from differences in weight of gauze bandages. The same numbers of bandages ( 3 pieces at once) were removed every 10 minutes during the first 30 minutes of the observation period and every 20 minutes thereafter to standardize the blood loss measurement protocol and to avoid overfilling
TABLE 2. In vitro coagulation assay

\begin{tabular}{lccc}
\hline & CU-2010 & CU-2020 & Aprotinin \\
\hline $\begin{array}{l}\text { Activated partial } \\
\text { thromboplastin time } \mathrm{EC}_{200}(\mu \mathrm{mol} / \mathrm{L})\end{array}$ & 1.4 & 32.8 & 11.9 \\
Prothrombin time $\mathrm{EC}_{200}(\mu \mathrm{mol} / \mathrm{L})$ & 9.1 & 36.7 & $>100$ \\
\hline
\end{tabular}

$E C_{200}$, Concentration required for 2-fold prolongation of clotting time.

of the operative area with blood. Secondary end points were parameters of coagulation (activated clotting time $[\mathrm{ACT}]$, prothrombin ratio (Quick method), and aPTT). These parameters were monitored before CPB and before application of heparin and study medications, at 45 and 90 minutes of $\mathrm{CPB}$, and after weaning from $\mathrm{CPB}$ at 105 and 220 minutes from initiation of CPB. ACT (celite method), prothrombin ratio, and PTT were assessed by routine clinical assays. Heart rate and arterial pressure were monitored continuously.

\section{Vascular Function}

Endothelium-dependent and -independent vasorelaxation responses were investigated in isolated coronary arterial rings from the dogs. After the end of the experiments, the coronary arteries were excised and placed in cold $\left(4^{\circ} \mathrm{C}\right) \mathrm{Krebs}-$ Henseleit solution $(118-\mathrm{mmol} / \mathrm{L}$ sodium chloride, 4.7- $\mathrm{mmol} / \mathrm{L}$ potassium chloride, $1.2-\mathrm{mmol} / \mathrm{L}$ potassium dihydrogen phosphate, $1.2-\mathrm{mmol} / \mathrm{L}$ magnesium sulfate, $1.77-\mathrm{mmol} / \mathrm{L}$ calcium chloride, $25-\mathrm{mmol} / \mathrm{L}$ sodium hydrogen carbonate, and $11.4-\mathrm{mmol} / \mathrm{L}$ glucose, $\mathrm{pH}$ 7.4). The coronary arteries were prepared, and cleansed of periadventitial fat and surrounding connective tissue, and cut transversely into 4-mm rings with an operation microscope. Rings from the left anterior descending and circumflex coronary arteries were used randomly.

Isolated coronary rings were mounted on stainless steel hooks in individual organ baths (Radnoti Glass Technology, Monrovia, Calif) containing 25 $\mathrm{mL}$ of Krebs-Henseleit solution at $37^{\circ} \mathrm{C}$ and aerated with $95 \%$ oxygen and $5 \%$ carbon dioxide. Special attention was paid during the preparation to avoid damaging the endothelium.

Isometric contractions were recorded with isometric force transducers (Radnoti Glass Technology). Data were digitized, stored, and displayed with the IOX Software System (EMKA Technologies, Paris, France).

The coronary rings were placed under a resting tension of $3.5 \mathrm{~g}$ and equilibrated for 60 minutes. During this period, tension was periodically adjusted to the desired level, and the Krebs-Henseleit solution was changed every 30 minutes. Potassium chloride was used in these experiments to prepare vessels for stable contractions and reproducible dose-response curves to other vasoactive agents. Coronary rings were contracted with $80-\mathrm{mmol} / \mathrm{L}$ potassium chloride and rinsed after the contraction until resting tension was again obtained. The thromboxane $\mathrm{A}_{2}$-receptor agonist U46619 $\left(5 \times 10^{-7}\right.$ $\mathrm{mol} / \mathrm{L}$ ) was used to contract the rings until a stable plateau was reached, and relaxation responses were examined by adding cumulative concentrations of the endothelium-dependent dilator acetylcholine $\left(10^{-9}-10^{-4} \mathrm{~mol} /\right.$ L) and the endothelium-independent dilator sodium nitroprusside (SNP, $10^{-10}-10^{-5} \mathrm{~mol} / \mathrm{L}$ ). Contractile responses are expressed as grams of tension, relaxation responses are expressed as percentages of contraction induced by U46619.

\section{Statistics}

All values are expressed as mean \pm SEM. The paired $t$ test was used to compare 2 means within groups. Individual means were compared between the groups by 1-way analysis of variance followed by an unpaired $t$ test with Bonferroni correction for multiple comparisons and the post hoc Scheffé test. In the figures, only the significant differences between groups are indicated. Significant changes with the time within each group are indicated in the text. 

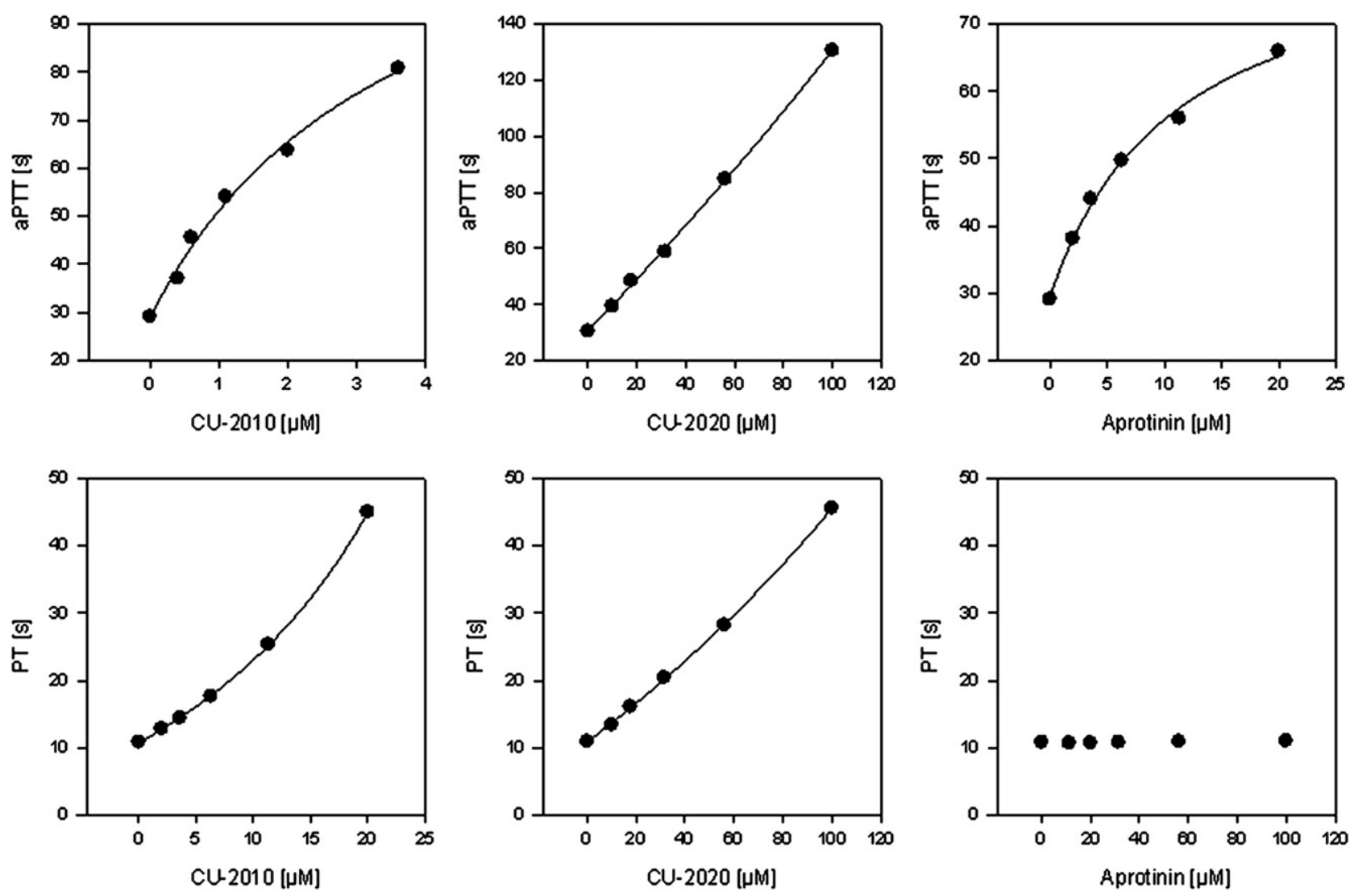

FIGURE 1. In vitro activated partial thromboplastin time $(a P T T)$ and prothrombin time $(P T)$ values. Dose-response relationships.

\section{RESULTS}

\section{In Vitro Measurements}

Table 1 shows $K_{i}$ values of the inhibition of human serine proteases. Plasmin inhibition was comparable between aprotinin and CU-2010 and CU-2020. Plasma kallikrein inhibition potency, however, was 30-fold improved with the analogs, as indicated by their markedly lower $\mathrm{K}_{\mathrm{i}}$ values. In addition, CU2010 and CU-2020 have considerable (as much as 100,000 times greater than aprotinin) inhibitory potential of factor $\mathrm{Xa}$ and factor XIa as well as thrombin. In vitro aPTT and corresponding $\mathrm{EC}_{200}$ values were similar for all 3 compounds. Although application of aprotinin does not influence PT values, there was a clear dose-dependent relationship when CU-2010 or CU-2020 was applied (Figure 1 and Table 2).

\section{Hemodynamics During CPB}

All animals were hemodynamically stable throughout the experiments. Hemodynamic variables did not differ either between the groups or with time. The time course of mean arterial pressure is shown in Figure 2.

\section{Blood Loss}

Postoperative blood loss during the first 2 hours after injection of protamine was significantly reduced with
CU-2010 and CU-2020 as well as with aprotinin relative to control $(P<.05)$. There were no differences between the 4 treatment groups (Figure 3).

\section{Blood Coagulation Parameters}

As expected, aPTT and ACT increased significantly in all groups after heparin and remained elevated during $\mathrm{CPB}$ (Figures 4 and 5). After protamine administration, ACT returned to baseline. Prothrombin ratio values decreased significantly during CPB and remained low until the end of the observation period (Figure 6). As shown in Figure 5, treatment with CU-2010 (continuous infusion scheme) and CU-2020 significantly elongated PTT after CPB at 105 and 220 minutes, respectively. There was also a tendency toward prolonged ACT values, although this trend did not reach the level of significance.

\section{Vascular Function}

Maximal isometric forces produced by the isolated coronary arterial rings contracted by the thromboxane $\mathrm{A}_{2}$-receptor agonist $\mathrm{U} 46619\left(5 \times 10^{-7} \mathrm{~mol} / \mathrm{L}\right)$ were not significantly altered in any of the experimental groups studied (data not shown). The endothelium-dependent, receptor-mediated vasorelaxation response of the precontracted coronary 


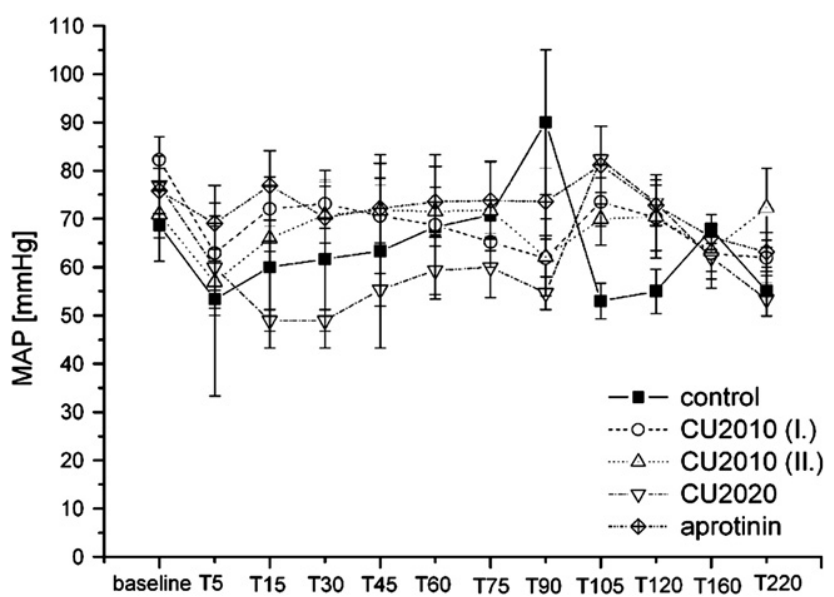

FIGURE 2. Time course of mean arterial pressure. All values are mean \pm SEM. Time points on $\mathrm{x}$-axis indicate time in minutes from initiation of cardiopulmonary bypass. CU2010 (I.), Hammersmith CU-2010 group; CU2010 (II.), continuous CU-2010 group.

arterial rings to acetylcholine was unaffected by all treatments scheme with synthetic aprotinin analogs (Figure 7). Application of the synthetic aprotinin analogs did not affect the endothelium- and receptor-independent cyclic guanosine monophosphate-mediated vasorelaxation response to SNP (Figure 8).

\section{DISCUSSION}

In this study, 2 novel synthetic small-molecule serine protease inhibitors significantly reduced postoperative blood loss after CPB comparably to aprotinin. In addition, neither aprotinin nor the novel serine protease inhibitors affected coronary endothelial function.

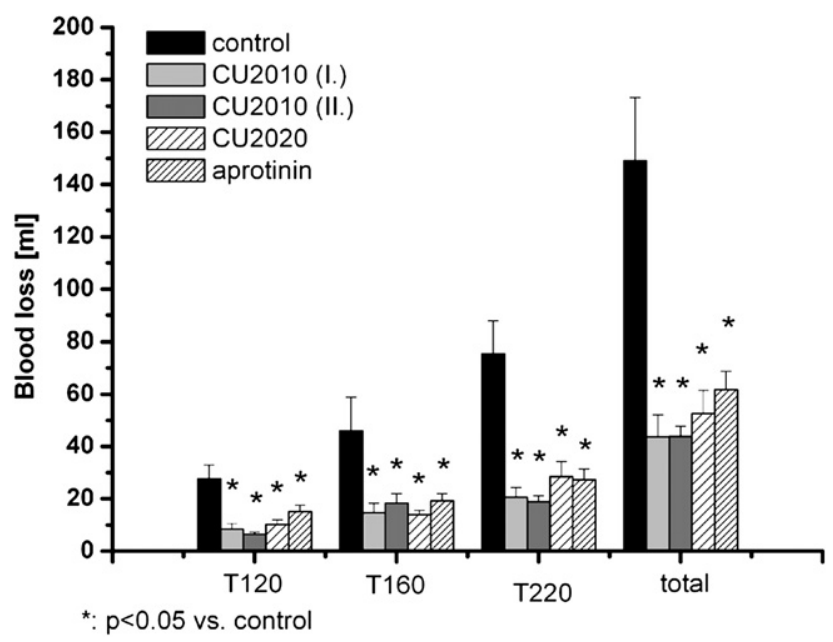

FIGURE 3. Blood loss after weaning from cardiopulmonary bypass and protamine. Time points indicate time in minutes from initiation of cardiopulmonary bypass, corresponding to 20 (T130), 60 (T160), and 120 (T220) minutes after protamine. All values are mean \pm SEM, Asterisk indicates $P<.05$ versus control. CU2010 (I.), Hammersmith CU-2010 group; CU2010 (II.), continuous CU-2010 group.

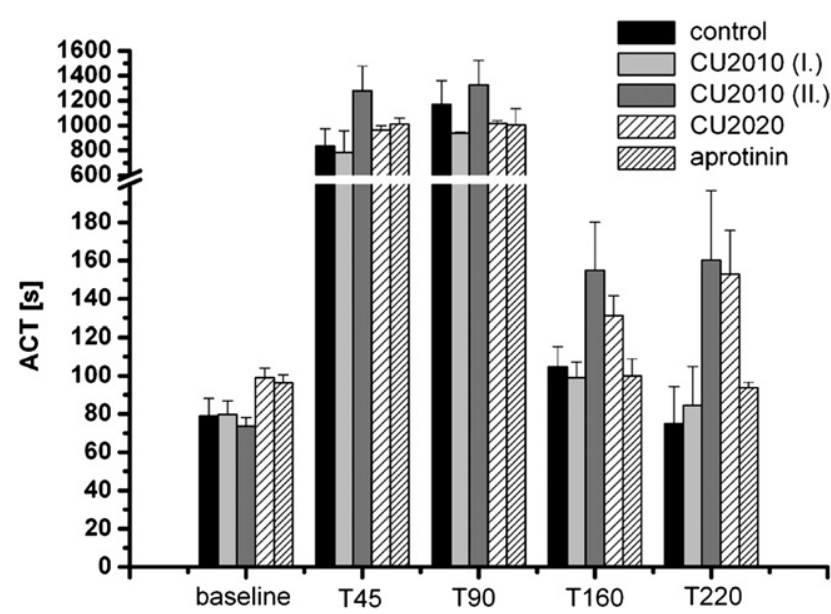

FIGURE 4. Activated clotting time (ACT). Time points indicate time in minutes from initiation of cardiopulmonary bypass; $T 220$ correspond to directly and 2 hours after protamine, respectively. All values are mean \pm SEM. CU2010 (I.), Hammersmith CU-2010 group; CU2010 (II.), continuous CU-2010 group.

Apart from the concerns raised about possible side effects of aprotinin, there is an evidence that aprotinin reduces postoperative blood loss and transfusion requirements as much as $40 \%$ to $50 \%$ in different population of patients (first time, reoperative, patients with aspirin, etc) undergoing cardiac surgery with CPB. ${ }^{12-14}$ Recently, significantly reduced blood loss was reported after the use of aprotinin in patients undergoing off-pump coronary artery bypass grafting. ${ }^{15}$ This study demonstrates for the first time that members of a new class of synthetic small-molecule serine protease inhibitors (CU-2010 and CU-2020) significantly decrease postoperative total blood loss after CPB in a canine model.

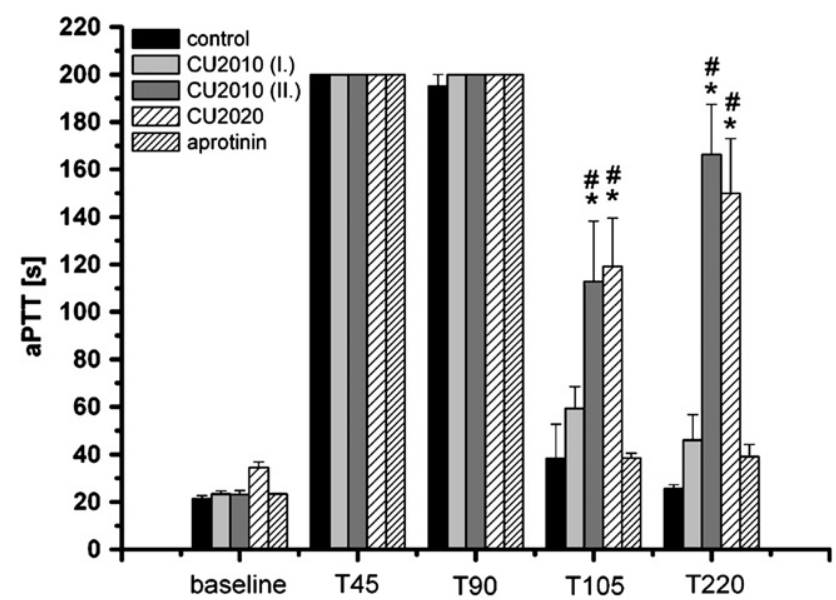

FIGURE 5. Activated partial thromboplastin time ( $a P T T)$. Time points indicate time in minutes from initiation of cardiopulmonary bypass; T105 and $T 220$ correspond to directly and 2 hours after protamine, respectively. All values are mean \pm SEM. Asterisk indicates $P<.05$ versus control; grid refers to $P<.05$ versus aprotinin. CU2010 (I.), Hammersmith CU-2010 group; CU2010 (II.), continuous CU-2010 group. 


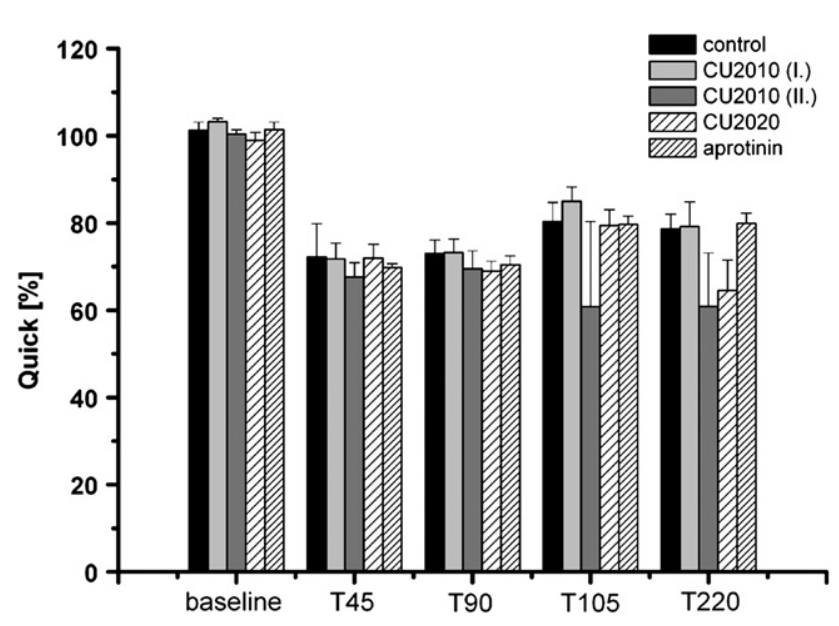

FIGURE 6. Prothrombin ratio (Quick method) expressed as percentage of reference plasma pool. Time points indicate time in minutes from initiation of cardiopulmonary bypass; T105 and T220 correspond to directly and 2 hours after protamine, respectively. All values are mean \pm SEM. CU2010 (I.), Hammersmith CU-2010 group; CU2010 (II.), continuous CU-2010 group.

The effects of CU-2010 and CU-2020 and aprotinin were not significantly different with the tested doses and application schemes.

These novel serine protease inhibitors may have some potential advantages, which may also overcome some of the concerns about the use of aprotinin that have led to its withdrawal from the market. First, in contrast to the biologic product aprotinin (a bovine peptide), CU-2010 and CU2020 are synthetic small molecules, and the well-known allergic reaction described after the use of aprotinin ${ }^{3}$ can therefore be reduced. The major cause of allergic reactions after application of the aprotinin product Trasylol was not

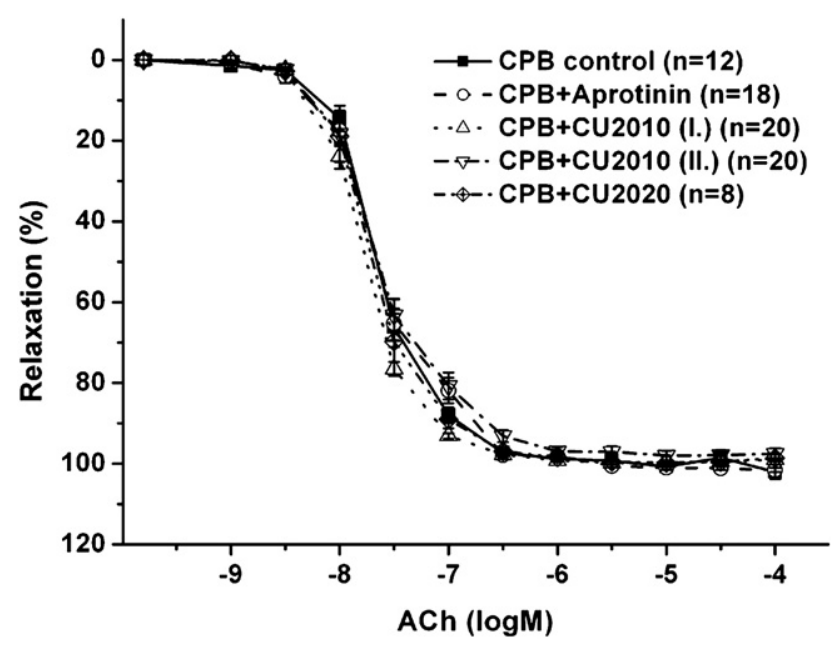

FIGURE 7. Endothelium-dependent relaxation after application of acetylcholine $(A C h)$. All values are mean \pm SEM. $C P B$, Cardiopulmonary bypass; CU2010 (I.), Hammersmith CU-2010 group; CU2010 (II.), continuous CU-2010 group.

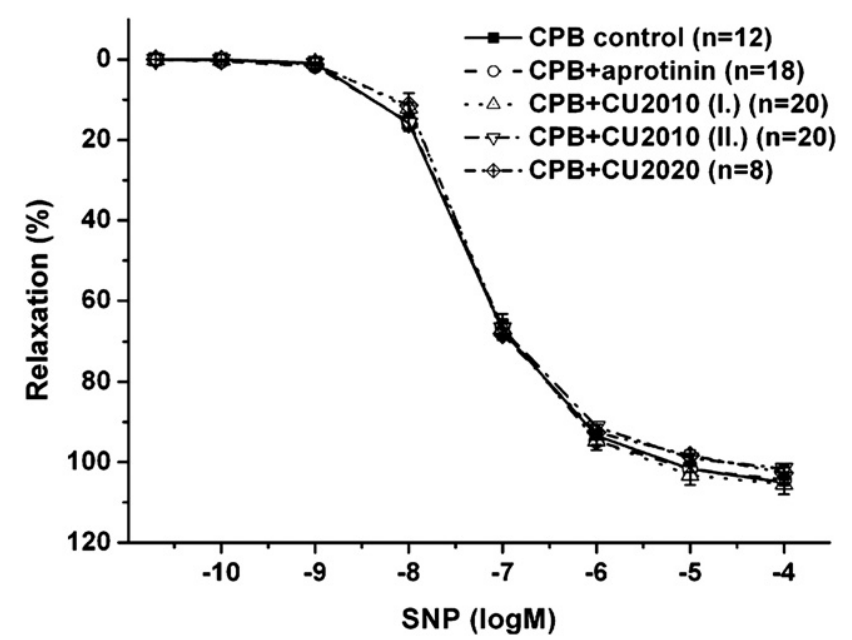

FIGURE 8. Endothelium-independent relaxation after application of sodium nitroprusside (SNP). CPB, Cardiopulmonary bypass; CU2010 (I.), Hammersmith CU-2010 group; CU2010 (II.), continuous CU-2010 group. All values are mean \pm SEM.

aprotinin itself but secondary bovine proteins and their fragments, which were still present in low concentrations because $100 \%$ purity could not be achieved during the manufacturing process from bovine lungs. These new compounds are thus less likely to be allergenic, because they are not natural products purified from an animal source.

Second, the novel protease inhibitors have a high antifibrinolytic potency complemented by moderate anticoagulatory properties. ${ }^{16}$ In contrast to aprotinin, CU-2010 and CU-2020 have additional factor $\mathrm{Xa}$ and stronger factor XIa inhibitory effects, which may protect against early thrombosis, one of the concerns in connection with the use of aprotinin. Indeed, in vitro tests have shown a significant dose-dependent prolongation of PT after application of CU-2010 and CU-2020, whereas aprotinin does not affect PT. In the our experimental model, only a somewhat lower prothrombin ratio (somewhat prolonged PT) was observed when CU-2010 was applied as a continuous infusion or after Hammersmith scheme application of CU-2020. It should be noted, however, that CPB and hemodilution probably had higher impacts on blood coagulation factors than did the factor Xa inhibitory effects of the test compounds themselves. Further investigations are necessary. Nevertheless, aPTT was still significantly prolonged after protamine in the CU-2010 continuous infusion and CU-2020 groups, whereas aPTT returned to baseline level in the other groups. This implies that the additional inhibitory potential against factors Xa and XIa provides an anticoagulatory potential and may have a protective effect against early thrombosis. It should be noted that even aprotinin may exert some antithrombotic effects. ${ }^{17,18}$

The observed differences in terms of blood coagulation parameters between the dosage schemes of CU-2010 can 
be explained by the very short half-life of this compound (17 minutes). After cessation of application of CU-2010, aPTT values normalized rapidly. If the infusion was continued until the end of the experiments, aPTT remained prolonged. The very short half-life of CU-2010 might be advantageous in allowing it to be used in an on-off switch mode, adapting the application to the actual hemostatic situation. CU-2020 has a longer half-life (45 minutes), thereby providing prolonged aPTT when applied by the Hammersmith scheme.

Measurement of ACT is a standard monitoring procedure for guidance of heparin-induced anticoagulation. Although the optimum ACT value for CPB has not been established, values between 400 and 480 seconds are commonly maintained. We applied the heparin doses according to the clinical calculations, which resulted in higher ACT values than in human beings. Nevertheless, application of protamine performed accordingly resulted in a rapid normalization of ACT levels in the control group. We therefore believe that higher ACT values during CPB did not influence blood loss after protamine significantly.

Initial investigations demonstrated that celite $\mathrm{ACT}$ values are prolonged by aprotinin. ${ }^{19}$ The increase in ACT is artificial caused by an in vitro interaction of the celite activator, heparin, and aprotinin. In contrast, aprotinin does not affect kaolin ACT. ${ }^{20}$ We used a celite-based ACT in our experiment, measured ACT values every 15 minutes, and maintained a value of more than 500 seconds during CPB. As expected, aPTT and ACT increased significantly in all groups after heparinization and remained elevated during CPB. It should be noted, however, that in the previously mentioned 2 treatment groups (continuous CU-2010, CU2020) ACT remained elevated in parallel with higher aPTT values after protamine. Further studies are necessary to determine whether the observed elevated ACT represents a true anticoagulant property.

Vascular and in particular endothelial function is an important determinant of functional recovery after cardiac surgery with $\mathrm{CPB}$ and early thrombus formation. In a recent report, ${ }^{21}$ we showed for the first time in a clinically relevant mammalian model of CPB that aprotinin has no significant effect on vascular function in epicardial coronary arteries. Previous investigations on the endothelial effects of aprotinin in other models have resulted in conflicting results. Ülker and coworkers ${ }^{10}$ found impaired endothelium-dependent vasorelaxation to acetylcholine and to the calcium ionophore A23187 in rat thoracic aortic rings and inhibition of bradykinin-induced endothelium-dependent coronary vasodilatation in coronary arteries of rat Langendorff-perfused hearts in the presence of aprotinin. ${ }^{11}$ In contrast to these findings, Khan and colleagues ${ }^{9}$ reported aprotinin-induced improvement of both endothelium-dependent and -independent vasorelaxation of coronary microvessels after regional ischemia and cardioplegic arrest in a porcine model. These data are in line with the results of Fischer and Steinhoff. ${ }^{22}$ With sub- stance $\mathrm{P}$ used to induce endothelium-dependent vasorelaxation, they found no significant effect of aprotinin at clinical dosage levels on endothelial function in porcine coronaries. ${ }^{22}$ Similar to our current results, endothelium-dependent relaxation to acetylcholine was unaffected by aprotinin treatment of human saphenous vein rings (explanted for coronary bypass) in a previous study. ${ }^{23}$ In this study, we have extended previous findings and showed that these 2 novel serine protease inhibitors do no have any detrimental effect on vascular relaxation, at least in vitro.

There have been only sporadic studies investigating the effect of aprotinin on endothelium-independent dilatory function of vascular smooth muscles. Most have reported no influence of aprotinin on endothelium-independent vasorelaxation to $\mathrm{SNP},{ }^{10,11}$ a nitric oxide donor substance that acts in a receptor-independent cyclic guanosine monophosphate-mediated manner by directly activating the guanylyl cyclase enzyme in vascular smooth muscle cells. Our current data completely correspond with these results; the SNP-induced vasorelaxation of coronary arterial rings was unaffected by aprotinin. Similarly, the novel serine protease inhibitors did not influence vascular smooth muscle function.

In conclusion, we have documented that the novel serine protease inhibitors CU-2010 and CU-2020 equivalently reduce blood loss and have improved anticoagulatory properties relative to aprotinin. Taking into account that these small synthetic molecules have numerous advantages relative to aprotinin, we believe that these substances may offer a true alternative in the postaprotinin era. Learning the lessons from the current debates, however, several safety trials should performed before moving into the clinical arena.

\section{References}

1. Dacey LJ, Munoz JJ, Baribeau YR, Johnson ER, Lahey SJ, Leavitt BJ, et al. Reexploration for hemorrhage following coronary artery bypass grafting. Arch Surg. 1998; $133: 442-7$

2. Keogh B, Kinsman R. Fifth national adult cardiac surgical database report 2003 Reading (UK): Dendrite Clinical Systems; 2004.

3. Beierlein W, Scheule AM, Dietrich W, Ziemer G. Forty years of clinical aprotinin use: a review of 124 hypersensitivity reactions. Ann Thorac Surg. 2005;79: 741-8.

4. Dietrich W, Spath P, Zuhlsdorf M, Dalichau H, Kirchhoff PG, Kuppe H, et al. Anaphylactic reactions to aprotinin reexposure in cardiac surgery: relation to antiaprotinin immunoglobulin G and E antibodies. Anesthesiology. 2001;95:64-71.

5. Katz BA, Sprengeler PA, Luong C, Verner E, Elrod K, Kirtley M, et al. Engineering inhibitors highly selective for the S1 sites of Ser190 trypsin-like serine protease drug targets. Chem Biol. 2001;8:1107-21.

6. Alderman EL, Levy JH, Rich JB, Nili M, Vidne B, Schaff H, et al. Analyses of coronary graft patency after aprotinin use: results from the International Multicenter Aprotinin Graft Patency Experience (IMAGE) trial. J Thorac Cardiovasc Surg. 1998;116:716-30.

7. Kalkat A, Levine, Dunning J. Does use of aprotinin in coronary artery bypass graft surgery affect graft patency? Interact Cardiovasc Thorac Surg. 2004;3:124-8.

8. Bidstrup BP, Underwood SR, Sapsford RN, Streets RM. Effect of aprotinin (Trasylol) on aorta-coronary bypass graft patency. J Thorac Cardiovasc Surg. 1993; 105:147-52

9. Khan TA, Bianchi C, Voisine O, Feng J, Baker J, Hart M, et al. Reduction of myocardial reperfusion injury by aprotinin after regional ischemia and cardioplegic arrest. J Thorac Cardiovasc Surg. 2004;128:602-8. 
10. Ülker S, Cinar MG, Bayraktutan U, Evinc A. Aprotinin impairs endothelium-dependent relaxation in rat aorta and inhibits nitric oxide release from rat coronary endothelial cells. Cardiovasc Res. 2001;50:589-96.

11. Ülker S, McKeown PP, Bayraktutan U. Aprotinin impairs coronary endothelial function and down-regulates endothelial NOS in rat coronary microvascular endothelial cells. Cardiovasc Res. 2002;55:830-7.

12. Blauhut B, Gross C, Necek S, Doran JE, Spath P, Lundsgaard-Hansen P. Effects of high-dose aprotinin on blood loss, platelet function, fibrinolysis, complement, and renal function after cardiopulmonary bypass. J Thorac Cardiovasc Surg. 1991;101:958-67.

13. Royston D, Bidstrup BP, Taylor KM, Sapsford RN. Effect of aprotinin on need for blood transfusion after repeat open-heart surgery. Lancet. 1987;2:1289-91.

14. Murkin JM, Lux J, Shannon NA, Guiraudon GM, Menkis AH, McKenzie FN, et al. Aprotinin significantly decreases bleeding and transfusion requirements in patients receiving aspirin and undergoing cardiac operations. J Thorac Cardiovasc Surg. 1994;107:554-61.

15. Bittner HB, Lamke J, Lange M, Rastan A, Mohr FW. The impact of aprotinin on blood loss and blood transfusion in off pump coronary artery bypass grafting. Ann Thorac Surg. 2008;85:1662-8.

16. Dietrich W, Nicklisch S, Koster A, Spannagl M, Giersiefen H, van de Locht A. CU-2010 - a novel small molecule protease inhibitor with antifibrinolytic and anticoagulant properties. Anesthesiology. 2009;110:123-30.

17. Landis RC, Asimakopoulos G, Poullis M, Haskard DO, Taylor KM. The antithrombotic and antiinflammatory mechanisms of action of aprotinin. Ann Thorac Surg. 2001;72:2169-75.

18. Khan TA, Bianchi C, Voisine P, Sandmeyer J, Feng J, Sellke FW. Aprotinin inhibits protease-dependent platelet aggregation and thrombosis. Ann Thorac Surg. 2005; 79:1545-50.

19. Despotis GJ, Filos KS, Levine V, Alsoufiev A, Spitznagel E. Aprotinin prolongs activated and nonactivated whole blood clotting time and potentiates the effect of heparin in vitro. Anesth Analg. 1996;82:1126-31.

20. Wang JS, Lin CY, Hung WT, Thisted RA, Karp RB. In vitro effects of aprotinin on activated clotting time measured with different activators. J Thorac Cardiovasc Surg. 1992;104:1135-40.

21. Veres G, Radovits T, Schulz H, Linn LN, Hütter J, Weigang E, et al. Effect of recombinant aprotinin on postoperative blood loss and coronary vascular function in a canine model of cardiopulmonary bypass. Eur J Cardiothorac Surg. 2007;28: $801-4$.

22. Fischer JH, Steinhoff M. Effects of aprotinin on endothelium-dependent relaxation of large coronary arteries. Eur J Cardiothorac Surg. 2005;28: 801-4.

23. Allen S, Anastasiou N, Royston D, Paniagua R, Yacoub M. Effect of aprotinin on vascular reactivity of coronary bypass grafts. J Thorac Cardiovasc Surg. 1997; 113:319-26.

\section{Discussion}

Dr Craig R. Smith (New York, NY). First, I thank you for sending me a manuscript a couple of weeks ago. That shows a high level of organization.

It's nice to bring compounds like this before us. It takes a lot of courage to try to do something like this when you know that this might work out very well but then 25 years from now somebody will publish a paper in the New England Journal of Medicine showing 100,000 patients in a meta-analysis with $0.2 \%$ increase in creatinine with a $P$ value of .00001 , and you'll be out of business.

You picked 2 drugs that are extremely potent as antikallikrein agents. In the $\mathrm{K}_{\mathrm{i}}$ tables, I gather you assume that the plasma $\mathrm{K}_{\mathrm{i}}$ completely summarizes the drugs' antifibrinolytic action. I'm not sure that's true, but perhaps it is. There may be other ways to directly assess antifibrinolysis, which has usually been assumed to be the main mechanism by which aprotinin reduces blood loss and has spawned other antifibrinolytic agents, which you're all familiar with, tranexamic acid and aminocaproic acid. Can you tell us anything about how your agents behave as antifibrinolytics, either with plasma $\mathrm{K}_{\mathrm{i}}$ or some more elaborate assessment of antifibrinolytic activity?

Dr Szabó. I only showed some examples of $\mathrm{K}_{\mathrm{i}}$ values on human serum proteases. And it's true that plasmin activation is not the only one mechanism in fibrinolysis.

We have also additional data where we additionally investigated the effects of these new compounds on fibrinolysis. And we have found, in terms of fibrinolysis, not really much difference from aprotinin; but then the aim of designing this model was to have something similar to aprotinin. What we did, and that was important to show, was try to add some other protease inhibitory actions in terms of improved anticoagulatory properties. As we showed in terms of factors $\mathrm{Xa}$ and XIa, there are considerable gulatory properties in these drugs.

Dr Smith. That's clearly an advantage of these drugs. But you didn't test their antifibrinolytic activity against tranexamic acid or other agents that are more specific?

Dr Szabó. No, just against aprotinin.

Dr Smith. Regarding my second question, I notice that you discussed celite in your manuscript. I'm still surprised that you chose to use a celite activator, knowing it sets you up for questions from somebody like me who will ask you about the celite-aprotinin interaction.

You assume, I gather, that the equivalence of ACTs in all the groups means that there was no artificial elevation of ACT by aprotinin, but can you tell us what the heparin doses were?

Dr Szabó. Well, in terms of celite ACT, I think that is an important issue in terms of management in the human situation. In our study, we could not document what we know from the clinical arena, that aprotinin artificially prolongs ACTs. Nevertheless, we used the normal human dose. It seems that dogs are more sensitive to heparin, so we have much higher ACT values, longer than 1000 or 1500 seconds, and that might be why we could not document the artificial prolongation of ACT in response to aprotinin. What we saw is that the new compounds also prolonged PTT, which was not prolonged by aprotinin. So we speculate that we have here a true prolongation of blood coagulation, and not an artificial situation, also in terms of ACT. This would imply that it in a human situation we would not need higher heparin doses for the patients.

Dr Smith. Since you didn't actually answer the question about what the heparin doses were in the different groups, I'll just move beyond that. But another way to look at that would be to look for evidence of thrombin generation in all the groups, for a host of reasons but one of them being to ensure that your ACTs were not inaccurately reflecting the degree of anticoagulation.

Dr Pirooz Eghtesady (Cincinnati, Ohio). Did you look at renal function or renal clearance of these drugs? Did you look at kidney function?

Dr Szabó. We have no data in this model about renal function. The only related measurement was the urinary output during CPB, primary with the aim of volume management, and we saw no differences between the groups. 